\title{
Schizophrenia-risk and urban birth are associated with proteomic changes in neonatal dried blood spots
}

\author{
Jason D. Cooper ${ }^{1}$, Sureyya Ozcan', Renee M. Gardner ${ }^{2}$, Nitin Rustogi', Susanne Wicks ${ }^{2,3}$, Geertje F. van Rees ${ }^{1}$,
} F. Markus Leweke ${ }^{4}$, Christina Dalman ${ }^{2,3}$, Håkan Karlsson $^{2}$ and Sabine Bahn ${ }^{1}$

\begin{abstract}
In the present study, we tested whether there were proteomic differences in blood between schizophrenia patients after the initial onset of the disorder and controls; and whether those differences were also present at birth among neonates who later developed schizophrenia compared to those without a psychiatric admission. We used multiple reaction monitoring mass spectrometry to quantify 77 proteins (147 peptides) in serum samples from 60 first-onset drug-naive schizophrenia patients and 77 controls, and 96 proteins (152 peptides) in 892 newborn blood-spot (NBS) samples collected between 1975 and 1985. Both serum and NBS studies showed significant alterations in protein levels. Serum results revealed that Haptoglobin and Plasma protease $\mathrm{C} 1$ inhibitor were significantly upregulated in first-onset schizophrenia patients (corrected $P<0.05$ ). Alpha-2-antiplasmin, Complement C4-A and Antithrombin-III were increased in first-onset schizophrenia patients (uncorrected $P$-values $0.041,0.036$ and 0.013 , respectively) and also increased in newborn babies who later develop schizophrenia ( $P$-values $0.0058,0.013$ and 0.044 , respectively). We also tested whether protein abundance at birth was associated with exposure to an urban environment during pregnancy and found highly significant proteomic differences at birth between urban and rural environments. The prediction model for urbanicity had excellent predictive performance in both discovery (area under the receiver operating characteristic curve $(A \cup C)=0.90)$ and validation $(A \cup C=0.89)$ sample sets. We hope that future biomarker studies based on stored NBS samples will identify prognostic disease indicators and targets for preventive measures for neurodevelopmental conditions, particularly those with onset during early childhood, such as autism spectrum disorder.
\end{abstract}

\section{Introduction}

Despite decades of research, the aetiology of schizophrenia is poorly understood. Schizophrenia is a severe and disabling psychiatric disorder involving impairments in perception, cognition and motivation that usually become evident in late adolescence or early adulthood.

\footnotetext{
Correspondence: Sabine Bahn (sb209@cam.ac.uk)

${ }^{1}$ Department of Chemical Engineering and Biotechnology, University of Cambridge, Cambridge, UK

${ }^{2}$ Department of Public Health Sciences, Karolinska Institutet, Stockholm, Sweden

Full list of author information is available at the end of the article

Jason D. Cooper and Sureyya Ozcan contributed equally to this work.

Håkan Karlsson and Sabine Bahn are joint senior authors.
}

Early diagnosis of schizophrenia is beneficial for patients as shorter periods of untreated psychosis have been linked to better patient outcomes ${ }^{1}$. However, as there are no diagnostic tests for schizophrenia, diagnosis is still based on the evaluation of signs and symptoms in clinical interviews. Consequently, misdiagnosis is common $^{2}$ as patients are required to acknowledge the occurrence of symptoms of psychosis, such as hallucinations and delusions. Furthermore, other psychiatric disorders can present with overlapping symptoms.

To date, most proteomic and biomarker studies have focused on the detection of changes in protein levels in 
patients with confirmed disease status versus healthy individuals. However, for certain adult onset diseases such as type 2 diabetes, hypertension and stroke, increasing attention is being given to detecting prognostic disease markers in early life and even in newborn babies ${ }^{3}$. Early detection of disease predisposition could allow for targeted prevention or amelioration of disease course before overt symptoms develop. This could be achieved by therapeutic or lifestyle interventions.

Since the late 1960s, newborn blood-spot (NBS) screening programs have become routine to test for rare but serious metabolic health conditions, such as cystic fibrosis and sickle cell disease. The stability of DNA, RNA, small molecules and proteins within the dried blood-spot (DBS), combined with the ease of collection, shipping and storage provide a powerful tool for screening programs and for large population-based surveys. DBS sampling will be particularly important for diseases like psychiatric disorders in which patient recruitment is notoriously difficult and expensive. $\mathrm{We}^{4}$ and other researchers ${ }^{5}$, have previously demonstrated the potential utility of DBS sampling for clinical proteomics and personalised medicine applications using multiple reaction monitoring (MRM). In MRM, a highly specific, reproducible and sensitive mass spectrometry (MS) technique, pre-defined protein peptides or small molecules of interest can be robustly quantified from small sample volumes.

Reported environmental risk factors for schizophrenia that potentially affect early neurodevelopment during pregnancy include infections ${ }^{6}$ and nutritional deficiencies $^{7}$, intrauterine growth restriction ${ }^{8}$ and other pregnancy and birth complications. Established risk factors following birth include infections ${ }^{6}$, socioeconomic and childhood adversity. Epidemiological studies have also revealed an increased risk of developing psychiatric disorders for individuals born ${ }^{9,10}$ and living ${ }^{11}$ in urban environments. However, whether the effect of urbanicity on schizophrenia incidence is a consequence of unknown risk factors associated with place of birth, place of residence or both is unclear.

In the present study, we tested whether serum protein abundance differed between first-onset drug-naive schizophrenia patients and controls. We then tested whether those protein differences were present in NBS samples collected from newborn babies who later developed schizophrenia ('future schizophrenia patients') and those without a psychiatric admission. For the latter analysis, we had to initially determine whether we could detect the targeted protein peptides in stored NBS samples collected from neonates born in Sweden between 1975 and 1985. As our study population included babies exposed to urban and rural environments during pregnancy, we also tested whether protein abundance at birth was associated with urbanicity.

\section{Materials and methods}

\section{Subjects}

The Cologne study, as previously described ${ }^{12,13}$, consisted of serum samples from 60 first-onset drug-naive schizophrenia patients and 79 age and sex matched controls recruited by the Department of Psychiatry, University of Cologne (Table 1a). The ethical committees of the Medical Faculty of the University of Cologne and Addenbrooke's Hospital (Cambridge, UK) approved the protocols of this study including procedures for sample collection and analysis. Informed consent was given in writing by all participants.

The Stockholm population, as previously described ${ }^{14}$, consisted of all persons (born in Sweden 1975-1985) and treated for non-affective psychosis within psychiatric services in Stockholm County as inpatients (from 1987) or outpatients (from 1997 until 2004). The other population consisted of persons born between 1975 and 1985 in two Northern counties (Västerbotten and Norrbotten) and treated for non-affective and other psychoses between 1987 and 2005. Control subjects had no history of inpatient psychiatric admission, according to the National Patient Register ${ }^{15}$, and had to be alive and resident in Sweden. The controls were matched for sex, birth year and birth hospital. The aim was to recruit two controls per patient. Schizophrenia was defined as ICD9-code 295 (excluding 295F and 295H) or ICD10-code F20. Nonaffective psychosis (excluding schizophrenia) was defined as ICD9-code 297, 298C-298X, 295F and 295H or ICD10code F21-F29. The Northern Sweden data also included affective psychosis patients defined as ICD9-code 296 and 299 or ICD10-code F39, F333, F323, F315, F312 and F302. We only formed a 'psychosis patient group' consisting of patients with either non-affective or affective psychosis as a validation sample set to assess whether the urban-rural associations identified in controls could also be detected in an independent cohort (i.e. the future disease status is not relevant for this comparison). At the end of December 2003, the neonatal study consisted of 645 controls (no psychiatric diagnosis, subsequently referred to as 'controls'), 172 psychosis patients and 75 schizophrenia patients (Table 1b). All samples were stored in the same NBS sample repository in Stockholm. We obtained the following information through linkage to the Medical Birth Register: ${ }^{16}$ gestational age at birth, birth weight and length, birth order, Apgar score, head circumference, maternal eclampsia, maternal immigration, maternal age and place of residency (municipality) at delivery. Data on population density (number of inhabitants per $\mathrm{km}^{2}$ ) for each municipality in 1991 was obtained from Statistics Sweden. The study was approved by the regional ethics committee in Stockholm and all participants provided their signed consent. 


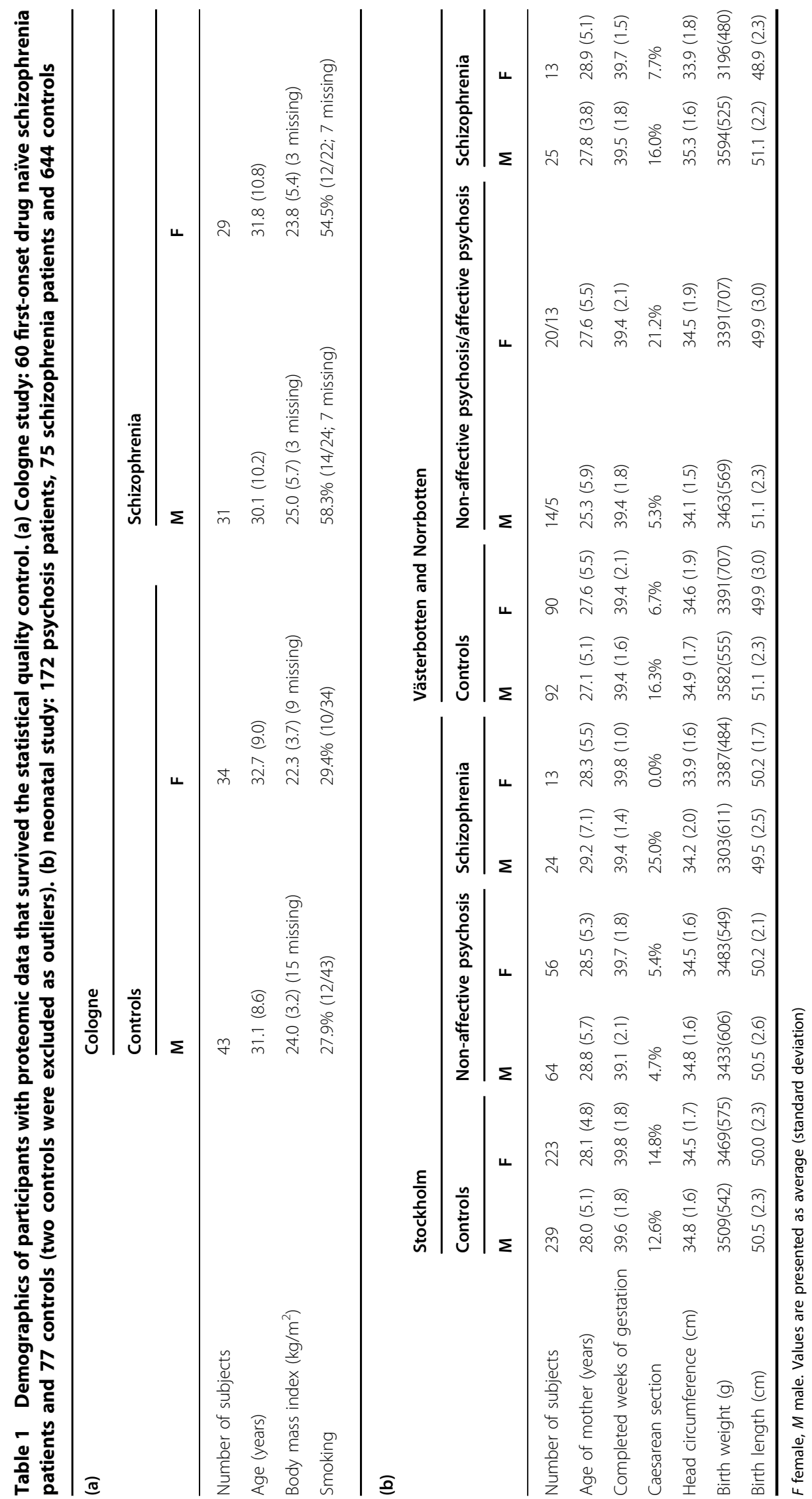




\section{Targeted protein quantification in NBS and serum}

NBS and serum samples were prepared in a 96 plate format as described previously ${ }^{4}$. Briefly, proteins were extracted from serum and NBS samples using ammonium bicarbonate. Then, disulphide bond reduction and cysteine alkylation were performed using dithiothreitol and iodoacetamide, respectively. Proteins digested overnight using trypsin (Supplementary Information). Isotopically labelled internal standard peptides were spiked into both NBS and serum samples prior to MS run. Quality control (QC) samples were used in this study to monitor method performance and instrument stability (Supplementary Information).

In this study, a total of 101 serum proteins (172 peptides), the majority previously associated with psychiatric disorders, were selected. Three to four interference-free transitions were selected for each targeted peptide as described previously ${ }^{4}$. Tryptic digested peptides were monitored using an Agilent 1290 Liquid Chromatography (LC) system coupled with 6495 Triple Quadrupole MS equipped with jet-stream nano ESI source operated in positive mode. MS data were acquired in MRM mode. The chromatographic separation was carried out on Agilent AdvanceBio Peptide Map column $(2.1 \times 150 \mathrm{~mm}$ 2.7-micron) at $50{ }^{\circ} \mathrm{C}$. Peptides were eluted over a linear gradient from $3 \%$ to $30 \%$ acetonitrile in $0.1 \%$ formic acid in $45 \mathrm{~min}$.

\section{Statistical analysis}

\section{Data pre-processing and quality control}

Raw MS files were processed using the Skyline software package (Version 3.1.0). Peaks were manually checked, and peak integrations were adjusted accordingly when necessary. The endogenous and internal standard peptide-transition peak areas were estimated and exported as a comma delimited data file for statistical analysis in $\mathrm{R}$ (Version 3.2.3) ${ }^{17}$. MS data pre-processing is described in the Supplementary Information.

\section{Coefficient of variation}

We used the geometric coefficient of variation (CV), which describes the amount of variability relative to the mean, to quantify the degree of variation for the peptides across the MS runs. For natural log transformed data, the geometric $\mathrm{CV}=\sqrt{e^{s d^{2}}-1} \times 100$ (ref. 18), where sd is the standard deviation of the log-transformed data. Note that the geometric CV was used as it is important to estimate the variability on the original scale of measurement.

\section{Patient-control association analysis}

We tested the association between relative peptide abundance and disease status ( 0 control and 1 schizophrenia) in the Cologne study using a logistic regression model. As body mass index was missing for over $20 \%$ of the participants and smoking for over $20 \%$ of patients (Table 1a), only age and sex were available for selection. In the analysis of schizophrenia patients and controls from the neonatal study, we used a generalised additive model $(\mathrm{GAM})^{19}$. As proteins dried on filter paper can degrade overtime ${ }^{20}$ and degradation may not be a linear function of time, we used a GAM to allow for a smooth to be fitted for year of birth, which represents the time of storage. The smooth may also better fit any changes in protein decay associated with the 1981 change in the storage of the Swedish NBS collection cards from room temperature to $4^{\circ}$ and $30 \%$ humidity. In the R package $\mathrm{mgcv}^{21}$, smooth functions of the GAM are represented using penalised regression splines. The following covariates were available for selection: sex, year of birth (linear or smooth; Supplementary Table 1), whether the mother was born abroad, Apgar score at $1 \mathrm{~min}$, Apgar score at 5 min, parity, whether the child was the first born, caesarean section, completed weeks of gestation, birth weight, length at birth, head circumference, whether the baby was small for their gestation age, age of mother, whether the mother suffered from eclampsia, and population density of the municipality where the mother was living at the time of the birth of the child (grouped as 0.1-49, 50-99, 100-499, 500-999, 1000-2999 and 3000-3999 per km²). We used the $\mathrm{R}$ package mice ${ }^{22}$ to replace missing covariate values using multiple-imputation (Supplementary Table 2). Model selection was based on forward-selection with Bayesian information criterion. We also fitted a joint effects model to predict disease status using ten-fold cross-validation with least absolute shrinkage and selection operator (lasso; Supplementary Information) regression as implemented in the R package glmnet ${ }^{23,24}$.

\section{Urban-rural association analysis}

We tested the association between relative peptide abundance and urbanicity at birth ( 0 rural and 1 urban) in controls from the neonatal study using a GAM. Rural was defined as a population density $<50$ per $\mathrm{km}^{2}$ and urban centre as a population density $\geq 1000$ per $\mathrm{km}^{2}$ (1500 per $\mathrm{km}^{2}$ used by European Union Organisation for Economic Co-operation and Development ${ }^{25}$ which falls within our population density group $1000-2999$ per $\mathrm{km}^{2}$ ), respectively containing 182 and 214 controls. Model selection, including lasso regression, and variables available for selection were as in the case-control comparison. We attempted to validate the urbanicity prediction model in 34 rural and 45 urban future (affective and non-affective) psychosis patients from the neonatal study. However, only location of birth and not future disease status were relevant for this comparison.

We measured the predictive performance of the diagnostic biomarker panel using sensitivity, specificity and area under the receiver operating characteristic curves 
(AUC: $0.9-1=$ excellent; $0.8-0.9=$ good; $0.7-0.8=$ fair; $0.6-0.7=$ poor; $\quad 0.5-0.6=$ fail). Optimal trade-offs between sensitivity and specificity were determined by maximising Youden's index $J$; where $J=$ sensitivity + specificity -1 .

\section{Results}

\section{Targeted protein detection and their coefficients of} variation

We monitored 77 proteins (147 peptides) in 139 serum samples from Cologne. These samples were randomly assigned to two 96-well plates, the second plate was half filled, and run over one and a half weeks on the MS. We used the CV to quantify the degree of variation (robustness) in the relative peptide abundances measured in a pooled serum sample. The median $\mathrm{CV}$ across the plates was $7.23 \%$ (6.54\% in plate 1 and $7.92 \%$ in plate 2; Supplementary Fig. 1a).

As we have previously only processed DBS samples within 6 months of collection ${ }^{4}$, we had to determine whether we could detect the targeted protein peptides in stored NBS samples collected between 1975 and 1985 (Supplementary Table 1). We initially tested ten samples collected in 1975 and 1985, five from each year. A total of 101 serum proteins were monitored in these test samples (data not shown) and 96 proteins (152 peptides) were selected and subsequently, monitored in 892 NBS samples. The samples were randomly assigned to ten 96-well plates and run over 10 weeks on the MS.

The median $\mathrm{CV}$ for the relative peptide abundances measured in a pooled NBS sample across plates $2-10$ was 10.83\% (range 9.50-11.52\%; Supplementary Fig. 1b), clearly demonstrating that we could reproducibly measure the targeted peptides in stored NBS samples collected between 1975 and 1985 .

\section{Patient and control analysis}

After QC, we analysed 68 proteins (128 peptides; Supplementary Table 3) in 60 first-onset drug-naive schizophrenia patients and 77 controls from Cologne. A total of 14 proteins (22 peptides) had an uncorrected $P<0.05$ for abundance differences between patients and controls (Table 2a). After $P$-values were corrected for multiple testing, three Haptoglobin (HPT) peptides and a Plasma Protease $\mathrm{C} 1$ Inhibitor (IC1) peptide were significant. The volcano plot suggested that there were more peptidetransitions with higher abundances in schizophrenia patients than would be expected by chance alone (Fig. 1a); which would result in a more symmetric pattern about around the $\log _{2}$ fold-change of zero. We note that only the apolipoproteins A2, A3, A4, C1 and C3 were downregulated in patients compared to controls (Table 2a; Fig. 1a). In total, 13 of these 14 proteins have previously been associated with schizophrenia (Table 4). Although IC1 has not been linked to schizophrenia before, recent reports have linked IC1 dysregulation to Alzheimer's disease ${ }^{26,27}$. The lasso prediction model consisted of 11 proteins (11 peptides; Supplementary Table 6a), and despite the absence of a clinical rating scale predictor, had a good predictive performance (area under the receiver operating characteristic curve $(\mathrm{AUC}=0.80)$ ).

We then investigated whether any of these 14 proteins (Table 2a) also differed in abundance at birth. To this end, we analysed NBS samples obtained from 75 future schizophrenia patients and 644 controls. In total, 12 of the 14 proteins were available for analysis. We found Alpha-2antiplasmin (A2AP), Complement C4-A (CO4A) and Antithrombin-III (ANT3) to be significantly different at birth (one-sided $P<0.05$; Table $2 \mathrm{~b}$ ) as well as after the onset of the disorder. We also analysed the remaining 73 proteins (110 peptides) measured in the neonatal study to investigate whether the abundance of any other proteins was significantly different at birth. No other proteins were significantly different between future schizophrenia patients and controls after $P$-values were corrected for multiple testing (Table 2c). As in the first-onset schizophrenia analysis (Fig. 1a), there were more peptidetransitions with higher abundances in future schizophrenia patients compared to controls (Fig. 1b).

\section{Urbanicity}

As birth in an urban environment has been associated with an increased risk for psychiatric disorders, we analysed 85 proteins (125 peptides) measured in 396 controls, 214 from urban and 182 from rural environments, to test whether protein abundance ratios at birth differed by urban-rural environment. Abundancies of 24 proteins (26 peptides) differed significantly after $P$-values were corrected for multiple testing and had a fold-change $>10 \%$ (Fig. 1c; Table 3). We then attempted to validate these 24 proteins using NBS from 79 psychosis patients, 45 from urban and 34 from rural environments. Fifteen of the 24 proteins were validated (16 peptides; one-sided $P<0.05$; Table 3). We did not attempt to further validate the associations in the future schizophrenia patients because of the relatively small number of patients from urban and rural environments, 17 and 38 respectively.

The lasso urbanicity prediction model, fitted to the controls, consisted of one covariate and 13 proteins (13 peptides; Supplementary Table $6 \mathrm{~b}$ ) and had an excellent predictive performance (AUC $=0.90$; Supplementary Fig. 2). We attempted to validate the fitted model in the psychosis patients and found that the excellent predictive performance was maintained (AUC $=0.89$; Supplementary Fig. 2).

The common functional pathways of the differentially expressed peptides listed in Tables 1 and 2 are summarised in Table 4. 
Table 2 (a) The most associated protein peptides with an uncorrected $P<0.05$ for the difference between 60 first-onset schizophrenia patients and 77 controls from the Cologne study. (b) The association for 12 of the 14 proteins reported in (a) and available in the 75 future schizophrenia patients and 644 controls from the neonatal study. (c) The most associated protein peptides with an uncorrected $P<0.10$ for the 75 future schizophrenia patients and 644 controls from the neonatal study

(a) Cologne study

\begin{tabular}{|c|c|c|c|c|}
\hline \multirow[t]{2}{*}{ Protein } & \multirow[t]{2}{*}{ Peptide } & \multicolumn{3}{|c|}{ First-onset schizophrenia and controls } \\
\hline & & Fold-change & $P$ & Corrected $P$ \\
\hline Haptoglobin (HPT) & VTSIQDWVQK & 1.54 & 0.000535 & 0.0283 \\
\hline Haptoglobin (HPT) & DYAEVGR & 1.56 & 0.000542 & 0.0283 \\
\hline Haptoglobin (HPT) & VGYVSGWGR & 1.53 & 0.000653 & 0.0283 \\
\hline Plasma protease $\mathrm{C} 1$ inhibitor (IC1) & TNLESILSYPK & 1.36 & 0.001480 & 0.0481 \\
\hline Apolipoprotein C-III (APOC3) & GWVTDGFSSLK & -1.25 & 0.00308 & 0.0801 \\
\hline Apolipoprotein A-IV (APOA4) & IDQNVEELK & -1.26 & 0.00829 & 0.172 \\
\hline Plasma protease C1 inhibitor (IC1) & FQPTLLTLPR & 1.34 & 0.0116 & 0.172 \\
\hline Apolipoprotein C-III (APOC3) & DALSSVQESQVAQQAR & -1.21 & 0.0120 & 0.172 \\
\hline Antithrombin-III (ANT3) & FDTISEK & 1.26 & 0.0121 & 0.172 \\
\hline Antithrombin-III (ANT3) & LPGIVAEGR & 1.25 & 0.0132 & 0.172 \\
\hline Complement C4-A (CO4A) & VLSLAQEQVGGSPEK & 1.24 & 0.0159 & 0.188 \\
\hline Alpha-1-antichymotrypsin (AACT) & EQLSLLDR & 1.38 & 0.0190 & 0.206 \\
\hline Apolipoprotein A-II (APOA2) & SPELQAEAK & -1.13 & 0.0211 & 0.209 \\
\hline Inter-alpha-trypsin inhibitor heavy chain $\mathrm{H} 4(\mathrm{ITIH} 4)$ & GPDVLTATVSGK & 1.18 & 0.0225 & 0.209 \\
\hline Complement component C9 (CO9) & WEESELAR & 1.23 & 0.0291 & 0.242 \\
\hline Apolipoprotein C-I (APOC1) & EFGNTLEDK & -1.26 & 0.0339 & 0.242 \\
\hline Complement component C9 (CO9) & LSPIYNLVPVK & 1.24 & 0.0354 & 0.242 \\
\hline Complement C4-A (CO4A) & ITQVLHFTK & 1.22 & 0.0357 & 0.242 \\
\hline Ficolin-3 (FCN3) & YGIDWASGR & 1.23 & 0.0364 & 0.242 \\
\hline Apolipoprotein A-IV (APOA4) & ISASAEELR & -1.21 & 0.0372 & 0.242 \\
\hline Alpha-2-antiplasmin (A2AP) & FDPSLTQR & 1.23 & 0.0412 & 0.255 \\
\hline Beta-2-glycoprotein 1 (APOH) & EHSSLAFWK & 1.26 & 0.0488 & 0.288 \\
\hline
\end{tabular}

(b) Neonatal study

\begin{tabular}{|c|c|c|c|c|}
\hline \multirow[t]{2}{*}{ Protein } & \multirow[t]{2}{*}{ Peptide } & \multicolumn{3}{|c|}{ Future schizophrenia and controls } \\
\hline & & Fold-change & $P$ & ${ }^{\text {a One-sided } P}$ \\
\hline Haptoglobin (HPT) & VTSIQDWVQK & - & - & - \\
\hline Haptoglobin (HPT) & DYAEVGR & - & - & - \\
\hline Haptoglobin (HPT) & VGYVSGWGR & - & - & - \\
\hline Plasma protease $\mathrm{C} 1$ inhibitor (IC1) & TNLESILSYPK & 1.001 & 0.9710 & - \\
\hline Apolipoprotein C-III (APOC3) & GWVTDGFSSLK & 1.002 & 0.9750 & - \\
\hline Apolipoprotein A-IV (APOA4) & IDQNVEELK & 1.123 & 0.0592 & b \\
\hline Plasma protease $\mathrm{C} 1$ inhibitor (IC1) & FQPTLLTLPR & - & - & - \\
\hline 'Apolipoprotein C-III (APOC3) & DALSSVQESQVAQQAR & 1.005 & 0.9200 & - \\
\hline Antithrombin-III (ANT3) & FDTISEK & - & - & - \\
\hline
\end{tabular}


Table 2 continued

\begin{tabular}{|c|c|c|c|c|}
\hline \multirow[t]{2}{*}{ Protein } & \multirow[t]{2}{*}{ Peptide } & \multicolumn{3}{|c|}{ Future schizophrenia and controls } \\
\hline & & Fold-change & $P$ & ${ }^{\text {a One-sided } P}$ \\
\hline Antithrombin-III (ANT3) & LPGIVAEGR & 1.092 & 0.0887 & 0.0444 \\
\hline Complement C4-A (CO4A) & VLSLAQEQVGGSPEK & 1.061 & 0.1860 & - \\
\hline cAlpha-1-antichymotrypsin (AACT) & EQLSLLDR & 1.062 & 0.2340 & - \\
\hline 'Apolipoprotein A-II (APOA2) & SPELQAEAK & 1.120 & 0.0397 & b \\
\hline Inter-alpha-trypsin inhibitor heavy chain $\mathrm{H} 4(\mathrm{ITIH} 4)$ & GPDVLTATVSGK & 1.015 & 0.7250 & - \\
\hline Complement component C9 (CO9) & WEESELAR & - & - & - \\
\hline Apolipoprotein C-I (APOC1) & EFGNTLEDK & 1.030 & 0.5260 & - \\
\hline 'Complement component C9 (CO9) & LSPIYNLVPVK & 1.003 & 0.9700 & - \\
\hline${ }^{\circ}$ Complement C4-A (CO4A) & ITQVLHFTK & 1.105 & 0.0267 & 0.01340 \\
\hline Ficolin-3 (FCN3) & YGIDWASGR & - & - & - \\
\hline Apolipoprotein A-IV (APOA4) & ISASAEELR & 1.087 & 0.1180 & - \\
\hline Alpha-2-antiplasmin (A2AP) & FDPSLTQR & 1.107 & 0.0116 & 0.00580 \\
\hline Beta-2-glycoprotein 1 (APOH) & EHSSLAFWK & 1.073 & 0.2990 & - \\
\hline
\end{tabular}

\section{(c) Neonatal study}

\begin{tabular}{|c|c|c|c|}
\hline \multirow[t]{2}{*}{ Protein } & \multirow[t]{2}{*}{ Peptide } & \multicolumn{2}{|c|}{ Future schizophrenia and controls } \\
\hline & & Fold-change & $P$ \\
\hline Transthyretin (TTHY) & VLDAVR & 1.156 & 0.00264 \\
\hline Alpha-2-antiplasmin (A2AP) & FDPSLTQR & 1.1074 & 0.0116 \\
\hline Protein AMBP (AMBP) & ETLLQDFR & 1.1102 & 0.0129 \\
\hline Serotransferrin (TRFE) & EGYYGYTGAFR & 1.1307 & 0.0166 \\
\hline C4b-binding protein alpha chain (C4BPA) & YTCLPGYVR & -1.1866 & 0.0204 \\
\hline Complement C4-A (CO4A) & ITQVLHFTK & 1.1055 & 0.0267 \\
\hline Tubulin alpha-4A chain (TBA4A) & EIIDPVLDR & 1.1094 & 0.0392 \\
\hline Apolipoprotein A-II (APOA2) & SPELQAEAK & 1.1203 & 0.0397 \\
\hline Clusterin (CLUS) & IDSLLENDR & 1.0977 & 0.0413 \\
\hline Ig gamma-3 chain C region (IGHG3) & DTLMISR & 1.0869 & 0.0418 \\
\hline Kininogen-1 (KNG1) & DFVQPPTK & 1.0769 & 0.0529 \\
\hline Ig gamma-3 chain C region (IGHG3) & NQVSLTCLVK & 1.0967 & 0.0534 \\
\hline Apolipoprotein A-IV (APOA4) & IDQNVEELK & 1.1225 & 0.0592 \\
\hline Apolipoprotein D (APOD) & VLNQELR & 1.0961 & 0.0643 \\
\hline Purine nucleoside phosphorylase (PNPH) & VFGFSLITNK & -1.0679 & 0.069 \\
\hline Apolipoprotein A-I (APOA1) & ATEHLSTLSEK & 1.0885 & 0.073 \\
\hline Alpha-2-antiplasmin (A2AP) & DFLQSLK & 1.0677 & 0.0844 \\
\hline Antithrombin-III (ANT3) & LPGIVAEGR & 1.0918 & 0.0887 \\
\hline Histone H4 (H4) & DAVTYTEHAK & 1.1005 & 0.0894 \\
\hline
\end{tabular}

$P$-values were corrected for multiple testing using the false discovery rate. The selected covariates are listed in Supplementary Table $4 a$. The table also includes APOA4 (IDQNVEELK), ANT3 (LPGIVAEGR), APOA2 (SPELQAEK), CO4A (ITQVLHFTK) and A2P2 (FDPSLTQR) from (b). The selected covariates are listed in Supplementary Table 4b. ${ }^{a}$ One-sided test conducted when the direction of the fold-change is consistent with that from the Cologne study and the two-sided $P<0.10$. ${ }^{b}$ Two-sided $P<0.10$, but direction of the fold-change is not consistent. 'Same protein peptide but different transition in (b) compared to (a) 
Cooper et al. Translational Psychiatry (2017)7:1290

Page 8 of 14

Discussion

We have previously demonstrated that we can successfully detect and reproducibly monitor tens of proteins isolated from serum and $\mathrm{DBS}^{4}$ samples using MRM, and here, we demonstrate that we can also do this in stored NBS samples collected between 1975 and 1985 (median (a)

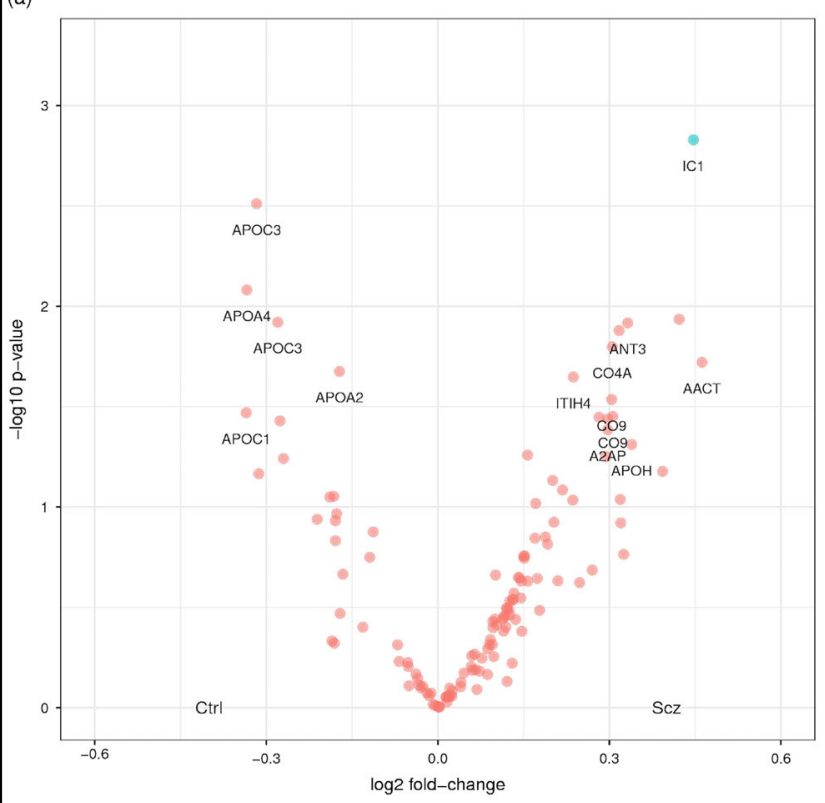

(c)

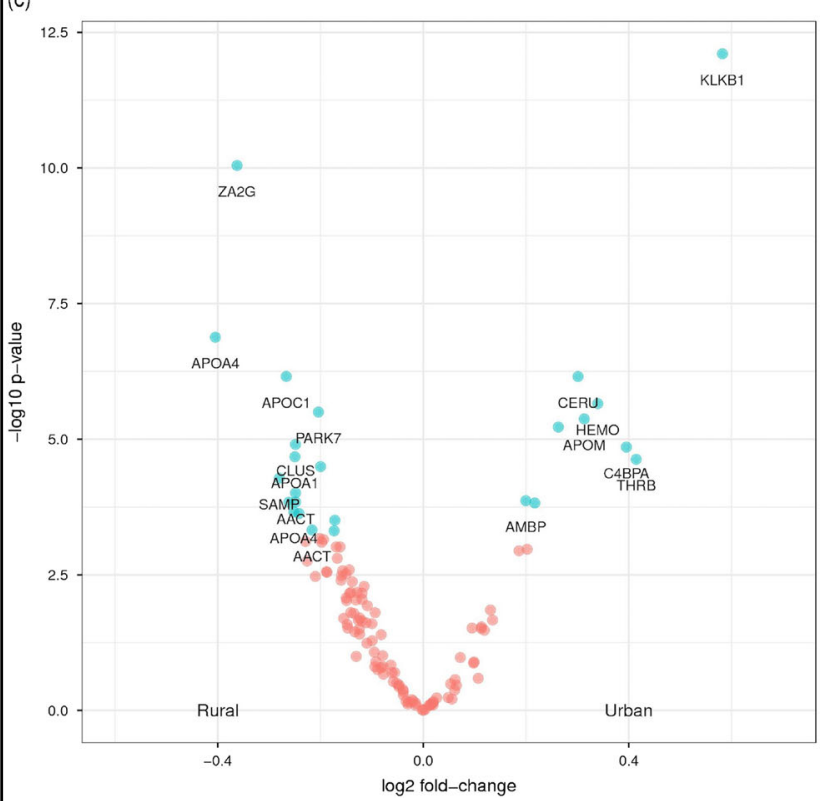

(b)

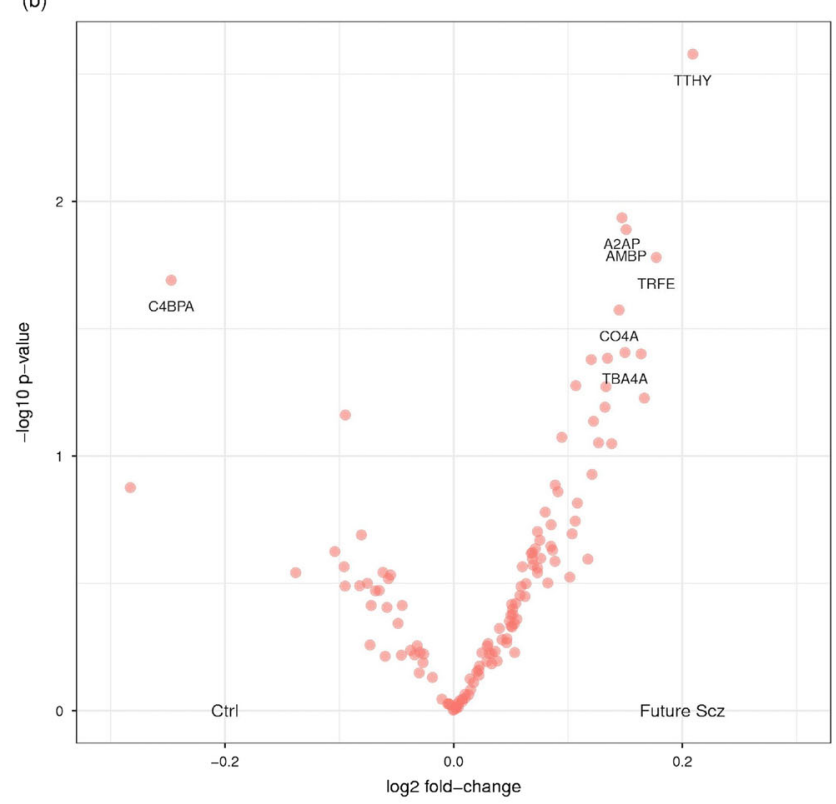

Fig. 1 a A volcano plot summarising the association between protein abundance in 60 first-onset schizophrenia patients and 77 controls from Cologne (Table aa). Light blue points indicate proteins that were significant after correction for multiple testing using the false discovery rate and had a fold-change $>10 \%$. Labelled proteins had uncorrected $P<0.05$. b A volcano plot summarising the association between protein abundance in 75 future schizophrenia patients and 644 controls from the neonatal study (Table Lc). Note that none of the protein changes remained significant after correction for multiple testing using the false discovery rate. Labelled proteins had uncorrected $P<0.05$. Interestingly, we identified a significantly greater number of increased proteins in the blood of newborn babies who were later diagnosed with schizophrenia. $\mathbf{c}$ A volcano plot summarising the association between protein abundance in urban and rural environments at birth. Note that serum albumin (ALBU) was excluded for display purposes only (Table 3). Light blue points indicate proteins that were significant after correction for multiple testing using the false discovery rate and had a fold-change $>10 \%$. Curl control, Sc schizophrenia 
Table 3 Peptide-transitions significantly associated with urban-rural environment at birth

\begin{tabular}{|c|c|c|c|c|c|c|}
\hline \multirow[t]{2}{*}{ Protein } & \multirow[t]{2}{*}{ Peptide } & \multicolumn{3}{|l|}{ Controls } & \multicolumn{2}{|l|}{ Psychosis } \\
\hline & & Fold-change & $P$ & Corrected $P$ & Fold-change & One-sided $\mathrm{P}$ \\
\hline Serum albumin (ALBU) & ETYGEMADCCAK & 3.220 & 3.79E-29 & $3.15 \mathrm{E}-27$ & 4.050 & 7.29E-10 \\
\hline Plasma kallikrein (KLKB1) & LSMDGSPTR & 1.497 & $7.85 \mathrm{E}-13$ & $6.52 \mathrm{E}-11$ & 1.818 & 1.19E-06 \\
\hline Zinc-alpha-2-glycoprotein (ZA2G) & AGEVQEPELR & -1.286 & $9.00 \mathrm{E}-11$ & 7.47E-09 & -1.211 & 0.0117 \\
\hline Apolipoprotein A-IV (APOA4) & IDQNVEELK & -1.324 & $1.32 \mathrm{E}-07$ & 1.10E-05 & -1.254 & 0.0143 \\
\hline Apolipoprotein C-I (APOC1) & EFGNTLEDK & -1.203 & $6.98 \mathrm{E}-07$ & $5.79 \mathrm{E}-05$ & -1.216 & 0.0147 \\
\hline Ceruloplasmin (CERU) & EVGPTNADPVCLAK & 1.232 & $7.00 \mathrm{E}-07$ & $5.81 \mathrm{E}-05$ & 1.222 & 0.0129 \\
\hline Hemopexin (HEMO) & VDGALCMEK & 1.265 & $2.21 \mathrm{E}-06$ & 0.00018343 & 1.086 & 0.268 \\
\hline Protein deglycase DJ-1 (PARK7) & DGLILTSR & -1.152 & $3.16 \mathrm{E}-06$ & 0.00026228 & -1.135 & 0.0345 \\
\hline Apolipoprotein M (APOM) & SLTSCLDSK & 1.243 & 4.23E-06 & 0.00035109 & 1.356 & 0.000394 \\
\hline Fructose-bisphosphate aldolase A (ALDOA) & ALANSLACQGK & 1.200 & $5.98 \mathrm{E}-06$ & 0.00049634 & 1.271 & 0.00479 \\
\hline Clusterin (CLUS) & IDSLLENDR & -1.188 & $1.25 \mathrm{E}-05$ & 0.0010375 & -1.233 & 0.0122 \\
\hline C4b-binding protein alpha chain (C4BPA) & YTCLPGYVR & 1.315 & $1.40 \mathrm{E}-05$ & 0.001162 & -1.015 & a \\
\hline Apolipoprotein A-I (APOA1) & ATEHLSTLSEK & -1.189 & $2.11 \mathrm{E}-05$ & 0.0017513 & -1.009 & 0.462 \\
\hline Prothrombin (THRB) & SGIECQLWR & 1.333 & $2.35 \mathrm{E}-05$ & 0.0019505 & 1.356 & 0.00339 \\
\hline Apolipoprotein E (APOE) & AATVGSLAGQPLQER & -1.148 & $3.20 \mathrm{E}-05$ & 0.002656 & -1.019 & 0.385 \\
\hline Serum amyloid P-component (SAMP) & IVLGQEQDSYGGK & -1.214 & $5.24 \mathrm{E}-05$ & 0.0043492 & -1.161 & 0.0623 \\
\hline Alpha-1-antichymotrypsin (AACT) & EQLSLLDR & -1.188 & 9.81E-05 & 0.0081423 & -1.237 & 0.0104 \\
\hline Protein AMBP (AMBP) & TVAACNLPIVR & 1.148 & 0.000136 & 0.011288 & 1.118 & 0.0581 \\
\hline Protein S100-A6 (S10A6) & LQDAEIAR & -1.188 & 0.000144 & 0.011952 & -1.015 & 0.438 \\
\hline Apolipoprotein A-II (APOA2) & SPELQAEAK & -1.199 & 0.000145 & 0.012035 & -1.06 & 0.288 \\
\hline Ig gamma-3 chain $\mathrm{C}$ region (IGHG3) & NQVSLTCLVK & 1.162 & 0.00015 & 0.01245 & 1.240 & 0.00564 \\
\hline Apolipoprotein A-IV (APOA4) & ALVQQMEQLR & -1.191 & 0.000219 & 0.018177 & -1.229 & 0.0153 \\
\hline Antithrombin-III (ANT3) & LPGIVAEGR & -1.182 & 0.000235 & 0.019505 & -1.206 & 0.0157 \\
\hline Phosphoglycerate kinase 1 (PGK1) & AGGFLMK & -1.127 & 0.000312 & 0.025896 & -1.074 & 0.179 \\
\hline Alpha-1-antichymotrypsin (AACT) & EIGELYLPK & -1.162 & 0.000473 & 0.039259 & -1.218 & 0.0116 \\
\hline Inter-alpha-trypsin inhibitor heavy chain $\mathrm{H} 2(\mathrm{ITIH} 2)$ & FYNQVSTPLLR & -1.128 & 0.000489 & 0.040587 & -1.090 & 0.141 \\
\hline
\end{tabular}

$P$-values were corrected for multiple testing using the false discovery rate. The selected covariates are listed in Supplementary Table $5 .{ }^{\text {a }}$ The direction of the foldchange in psychosis patients was not consistent with that in control subjects and so a one-sided test was not conducted (two-sided $P=0.00116$ )

CV 10.8\%; Supplementary Fig. 2). This has important research implications for countries that routinely store NBS samples and have an associated patient registry (such as Sweden and other Nordic countries) because of the potential to identify prognostic markers for conditions with an onset during early childhood, such as autism, attention deficit hyperactivity disorder and certain types of epilepsy.

We identified serum proteins that differ between firstonset drug-naive patients and controls (Table 2a), 13 of which have previously been associated with schizophrenia (Table 4). We also tested whether any of these proteins were significantly different in NBS samples from newborn babies who later developed schizophrenia and those without a psychiatric diagnosis. The levels of A2AP, CO4A and ANT3 were found to be significantly different at birth (Table 2b). Both A2AP and ANT3 are protease inhibitors, regulating a wide variety of biological processes including coagulation and inflammation and are involved in oxidative stress responses ${ }^{28-30}$.

Genetic variants associated with greater expression of $\mathrm{CO} 4 \mathrm{~A}$, a split product of $\mathrm{C} 4$, have previously been associated with an increased risk of schizophrenia ${ }^{31}$. The classic complement cascade, of which $\mathrm{C} 4$ is a member, is 


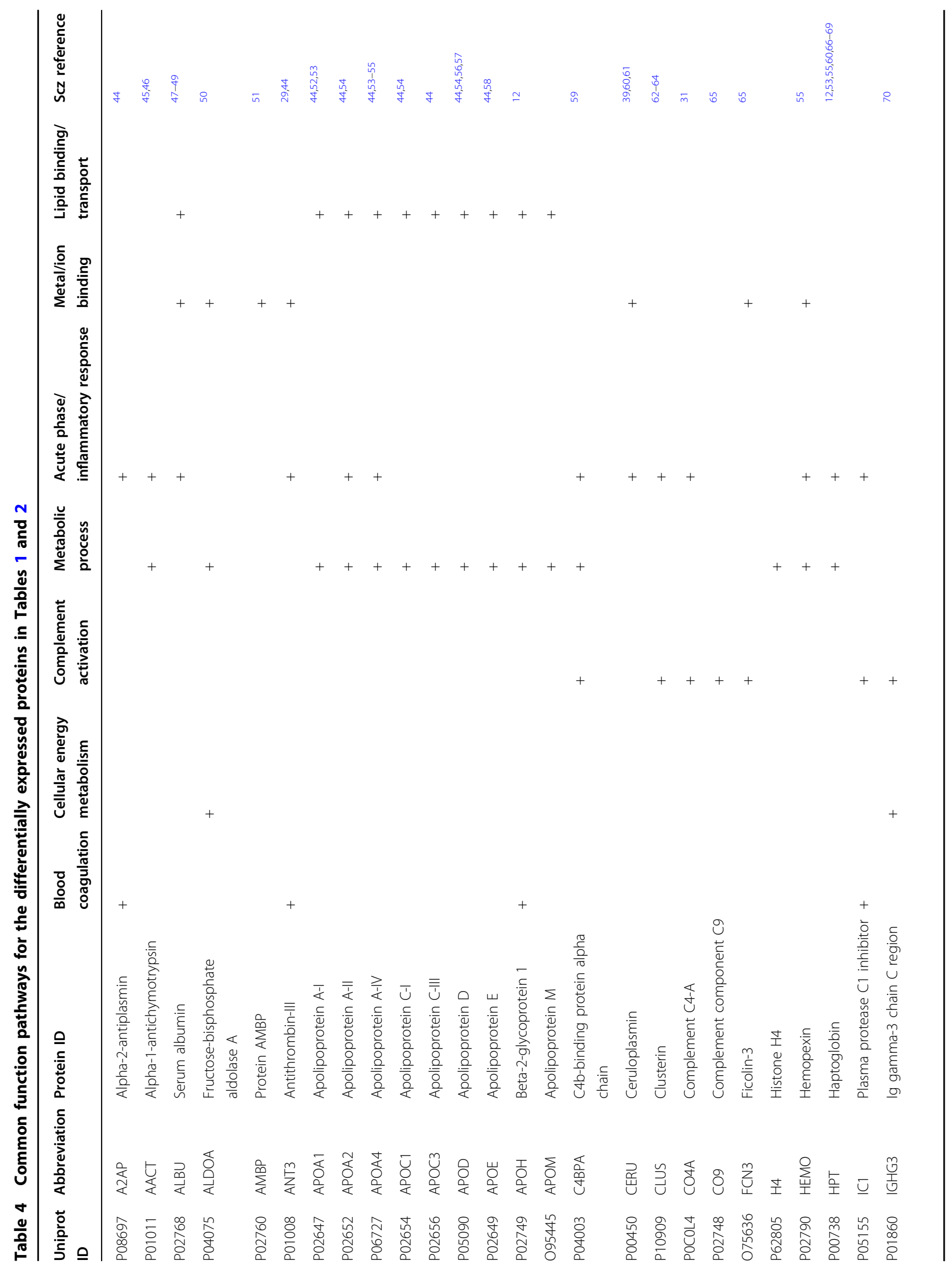




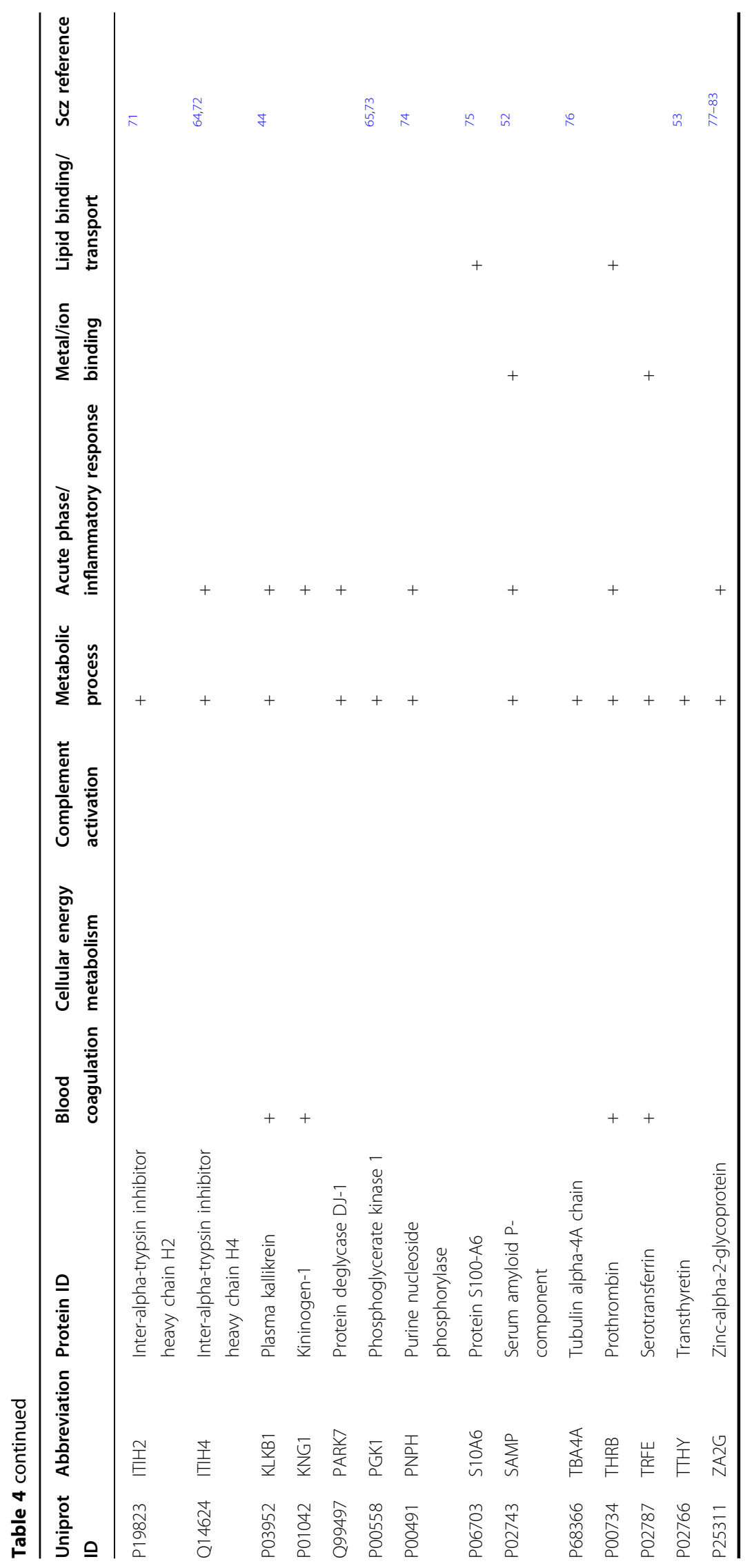


critically involved in synaptic pruning processes ${ }^{32}$. In the immune system, $\mathrm{C} 4$ promotes the activation of complement component $\mathrm{C} 3$, which in turn modulates inflammation processes in blood. Interestingly, studies in mice indicate that $\mathrm{C} 4$ can also mediate synapse elimination during postnatal development ${ }^{31-33}$. In support of our current findings, Hakobyan et al. ${ }^{34}$ previously reported elevated $\mathrm{C} 4$ activity in serum from individuals diagnosed with schizophrenia as compared to controls. Futhermore, the volcano plot (Fig. 1b) of the neonatal study results suggest a greater number of proteins with increased levels in the blood of newborn babies who were later diagnosed with schizophrenia than would be expected by chance alone; suggesting an increase in several inflammationrelated proteins, as previously reported for adult firstonset schizophrenia patients ${ }^{12}$ and evident in the Cologne study (Fig. 1a).

Although the pathogenesis of schizophrenia remains unknown, increasing evidence from genomic, transcriptomic and proteomic studies supports a role for coagulation, metabolism and inflammation ${ }^{35-39}$. Other predisposing factors include ethnicity, lifestyle, pre-natal and neonatal infections, maternal malnutrition and complications during birth. A common pathological pathway for these predisposing factors could be their common propensity to induce cellular metabolic stress which increase the possibility of oxidative stress and damage ${ }^{40}$. Our findings could suggest that an increased oxidative stress response may represent an inherent schizophrenia vulnerability.

As birth in an urban environment has been associated with an increased risk for psychiatric disorders ${ }^{10}$, we tested whether protein abundance at birth was associated with urbanicity. We found 24 proteins significantly associated with urbanicity in 397 controls (214 urban and 183 rural; Table 3) and confirmed 15 of the 24 proteins in a validation cohort of 97 psychosis patients (45 urban and 52 rural; Table 3 ). The majority of these 15 differentially expressed proteins relate to immune, especially the acute phase response, and metabolic function (Table 4). The protein with the greatest fold-change was albumin, 3.2fold and 4.1-fold in controls and psychosis patients, respectively. This is of interest, as albumin has been shown to be the main plasma protein in newborn babies which is modified by oxidative stress, especially through non-bound plasma metals such as iron ${ }^{41}$. Furthermore, several calcium and copper binding proteins were found to differ between urban and rural birth environments, notably ceruloplasmin (CERU; Tables 2 and 3). CERU is a major copper binding protein in plasma ${ }^{42}$ and has previously been associated with neuropsychiatric diseases including schizophrenia ${ }^{43-47}$. Interestingly, altered levels of CERU has also been linked to Wilson's disease which can present with schizophrenia-like psychosis and can result in misdiagnosis ${ }^{48,49}$. An urban environment is not only associated with more stress and trauma, adverse lifestyles such as drug and alcohol problems among pregnant mothers, but also with air pollution ${ }^{50}$. Previous studies indicate that air pollution in urban locations can affect cognitive and brain development directly. Some air pollutants, such as lead, can cross the blood brain barrier resulting in immune dysregulation and oxidative stress responses at both systemic and brain levels ${ }^{50}$. The predictive performance of the lasso derived model for birth environment was excellent (AUC $=0.90$; Supplementary Fig. 2), and this was maintained when we applied the fitted model to the psychosis patient group $(\mathrm{AUC}=0.89$; Supplementary Fig. 2). Although the ability to distinguish between birth in urban and rural environments per se may not be clinically relevant, it is of great interest that oxidative stress-related protein changes could be identified in both newborn babies who later develop schizophrenia as well as in babies born in an urban setting. The newborn infant is very susceptible to oxidative damage and a wide variety of consumer products and industrial pollutants have been associated with neurotoxicity in distinct developmental time windows ${ }^{51}$. Antioxidant protection for pregnant mothers and newborn infants in the form of dietary supplementation could be evaluated in future epidemiological studies.

There are several limitations to the present study. First, the number of patients and controls from Cologne limit the statistical power of the analysis. Second, given the time lag between birth and schizophrenia diagnosis, relatively few differences in protein abundance were observed. The investigation of a larger number of individuals who later develop schizophrenia will be required to provide the statistical power to identify robust protein abundance differences at birth. Third, as none of the analysed studies have been genotyped, we cannot test whether genetic variants are also associated with elevated levels of $\mathrm{CO} 4 \mathrm{~A}$ abundance. Fourth, as population density is based on municipality, and some municipalities are geographically large and include smaller and densely populated areas, the indicator of urbanicity used here is crude.

In conclusion, we have demonstrated that reproducible multiplexed quantitation of proteins in stored NBS samples can be achieved using MRM. We have provided further evidence that A2AP, CO4A and ANT3 may be associated with schizophrenia risk and the early disease process. The CO4A association is of particular interest given that genetic variants in $\mathrm{CO} 4 \mathrm{~A}$ have previously been associated with schizophrenia risk and offer additional support for its potential role in the aetiology of schizophrenia. In addition, we found and validated proteomic differences associated with birth environment. Future biomarker studies based on stored NBS samples used in 
conjunction with MRM could have the potential to identify risk factors and/or early disease indicators for conditions with onset during early childhood.

\section{Acknowledgements}

This study was supported by the Stanley Medical Research Institute (SMRI) and the Swedish Research Council. We also thank Agilent Technologies, especially Anthony Sullivan and Richard Blankley, for their support and ongoing collaborations. We thank the serum donors and the Department of Psychiatry, University of Cologne (Germany), for the provision of biological samples, in addition to support staff at the affiliated institutions.

\section{Author details}

'Department of Chemical Engineering and Biotechnology, University of Cambridge, Cambridge, UK. ${ }^{2}$ Department of Public Health Sciences, Karolinska Institutet, Stockholm, Sweden. ${ }^{3}$ Centre for Epidemiology and Community Medicine, Stockholm County Council, Stockholm, Sweden. ${ }^{4}$ Brain and Mind Centre, University of Sydney, Sydney, Australia

\section{Competing interests}

S.B. is a director of Psynova Neurotech Ltd and PsyOmics Ltd.

\section{Publisher's note}

Springer Nature remains neutral with regard to jurisdictional claims in published maps and institutional affiliations.

\section{Supplementary Information}

The online version of this article (https://doi.org/10.1038/s41398-017-0027-0) contains supplementary material.

Received: 5 April 2017 Revised: 12 July 2017 Accepted: 20 August 2017 Published online: 18 December 2017

\section{References}

1. van der Gaag, M. et al. Preventing a first episode of psychosis: meta-analysis of randomized controlled prevention trials of 12 month and longer-term followups. Schizophr. Res. 149, 56-62 (2013).

2. Gonzalez-Pinto, A. et al. First episode in bipolar disorder: misdiagnosis and psychotic symptoms. J. Affect. Disord. 50, 41-44 (1998).

3. Rakyan, V. K., Down, T. A., Balding, D. J. \& Beck, S. Epigenome-wide association studies for common human diseases. Nat. Rev. Genet. 12, 529-541 (2011).

4. Ozcan, S. et al. Towards reproducible MRM based biomarker discovery using dried blood spots. Sci. Rep. 7, 45178 (2017).

5. Chambers, A. G., Percy, A. J., Yang, J., Camenzind, A. G. \& Borchers, C. H. Multiplexed quantitation of endogenous proteins in dried blood spots by multiple reaction monitoring-mass spectrometry. Mol. Cell. Proteom. 12, 781-791 (2013).

6. Blomstrom, A. et al. Associations between maternal infection during pregnancy, childhood infections, and the risk of subsequent psychotic disorder--A Swedish Cohort Study of nearly 2 million individuals. Schizophr. Bull. 42 , 125-133 (2016).

7. Mackay, E., Dalman, C., Karlsson, H. \& Gardner, R. M. Association of gestational weight gain and maternal body mass index in early pregnancy with risk for nonaffective psychosis in offspring. JAMA Psychiatry 74, 339-349 (2017).

8. Nielsen, P. R. et al. Fetal growth and schizophrenia: a nested case-control and case-sibling study. Schizophr. Bull. 39, 1337-1342 (2013).

9. Mortensen, P. B. et al. Effects of family history and place and season of birth on the risk of schizophrenia. N. Engl. J. Med. 340, 603-608 (1999).

10. Vassos, E., Agerbo, E., Mors, O. \& Pedersen, C. B. Urban-rural differences in incidence rates of psychiatric disorders in Denmark. Br. J. Psychiatry 208, 435-440 (2016).

11. Vassos, E., Pedersen, C. B., Murray, R. M., Collier, D. A. \& Lewis, C. M. Metaanalysis of the association of urbanicity with schizophrenia. Schizophr. Bull. $\mathbf{3 8}$ 1118-1123 (2012).
12. Chan, M. K. et al. Development of a blood-based molecular biomarker test for identification of schizophrenia before disease onset. Transl. Psychiatry 5, e601 (2015).

13. Huang, J. T. et al. Independent protein-profiling studies show a decrease in apolipoprotein A1 levels in schizophrenia CSF, brain and peripheral tissues. Mol. Psychiatry 13, 1118-1128 (2008).

14. Blomstrom, A. et al. Maternal antibodies to infectious agents and risk for nonaffective psychoses in the offspring--a matched case-control study. Schizophr. Res. 140, 25-30 (2012).

15. Ludvigsson, J. F. et al. External review and validation of the Swedish national inpatient register. BMC Public Health 11, 450 (2011).

16. National Board of Health and Welfare. The Swedish Medical Birth register: A Summary of Content and Quality. (National Board of Health and Welfare, Stockholm, Sweden, 2003).

17. R Core Team. R: A language and Environment for Statistical Computing. (R Foundation for Statistical Computing, Vienna, Austria, 2015).

18. Schiff, M. H., Jaffe, J. S. \& Freundlich, B. Head-to-head, randomised, crossover study of oral versus subcutaneous methotrexate in patients with rheumatoid arthritis: drug-exposure limitations of oral methotrexate at doses $>/=15 \mathrm{mg}$ may be overcome with subcutaneous administration. Ann. Rheum. Dis. 73, 1549-1551 (2014).

19. Hastie, T. \& Tibshirani, R. Generalized additive-models - some applications. J. Am. Stat. Assoc. 82, 371-386 (1987).

20. Bowen, C. L., Hemberger, M. D., Kehler, J. R. \& Evans, C. A. Utility of dried blood spot sampling and storage for increased stability of photosensitive compounds. Bioanalysis 2, 1823-1828 (2010).

21. Wood, S. N. Fast stable restricted maximum likelihood and marginal likelihood estimation of semiparametric generalized linear models. J. R. Stat. Soc. B 73, 3-36 (2011).

22. van Buuren, S. \& Groothuis-Oudshoorn, K. mice: Multivariate Imputation by Chained Equations in R. J. Stat. Softw. 45, 1-67 (2011).

23. Tibshirani, R. Regression shrinkage and selection via the Lasso. J. R. Stat. Soc. B Met. 58, 267-288 (1996).

24. Hastie T., Tibshirani R., Friedman J. The Elements of Statistical Learning: Data Mining, Inference, and Prediction 5th edn (Springer, New York, NY, USA, 2001).

25. OECD. Redefining "Urban". (OECD Publishing, Paris, France, 2012).

26. Chiam, J. T., Dobson, R. J., Kiddle, S. J. \& Sattlecker, M. Are blood-based protein biomarkers for Alzheimer's disease also involved in other brain disorders? A systematic review. J. Alzheimer's Dis. 43, 303-314 (2015).

27. Muenchhoff, J. et al. Changes in the plasma proteome at asymptomatic and symptomatic stages of autosomal dominant Alzheimer's disease. Sci. Rep. $\mathbf{6}$, 29078 (2016).

28. Rau, J. C., Beaulieu, L. M., Huntington, J. A. \& Church, F. C. Serpins in thrombosis, hemostasis and fibrinolysis. J. Thromb. Haemost. 5, 102-115 (2007).

29. Zhang, X. et al. Quantitative proteomic analysis of serum proteins in patients with Parkinson's disease using an isobaric tag for relative and absolute quantification labeling, two-dimensional liquid chromatography, and tandem mass spectrometry. Analyst 137, 490-495 (2012).

30. Fiorini, A. et al. Involvement of oxidative stress in occurrence of relapses in multiple sclerosis: the spectrum of oxidatively modified serum proteins detected by proteomics and redox proteomics analysis. PLOS. ONE 8, e65184 (2013).

31. Sekar, A. et al. Schizophrenia risk from complex variation of complement component 4. Nature 530, 177-183 (2016).

32. Stevens, B. et al. The classical complement cascade mediates CNS synapse elimination. Cell 131, 1164-1178 (2007).

33. Schafer, D. P. et al. Microglia sculpt postnatal neural circuits in an activity and complement-dependent manner. Neuron 74, 691-705 (2012).

34. Hakobyan, S., Boyajyan, A. \& Sim, R. B. Classical pathway complement activity in schizophrenia. Neurosci. Lett. 374, 35-37 (2005).

35. Hoirisch-Clapauch, S., Amaral, O. B., Mezzasalma, M. A., Panizzutti, R. \& Nardi, A. E. Dysfunction in the coagulation system and schizophrenia. Transl. Psychiatry 6, e704 (2016)

36. Carrizo, E. et al. Coagulation and inflammation markers during atypical or typical antipsychotic treatment in schizophrenia patients and drug-free firstdegree relatives. Schizophr. Res. 103, 83-93 (2008).

37. Gardner, R. M., Dalman, C., Wicks, S., Lee, B. K. \& Karlsson, H. Neonatal levels of acute phase proteins and later risk of non-affective psychosis. Transl. Psychiatry 3, e228 (2013) 
38. Prabakaran, S. et al. Mitochondrial dysfunction in schizophrenia: evidence for compromised brain metabolism and oxidative stress. Mol. Psychiatry 9 684-697 (2004).

39. Flatow, J., Buckley, P. \& Miller, B. J. Meta-analysis of oxidative stress in schizophrenia. Biol. Psychiatry 74, 400-409 (2013).

40. Emiliani, F. E., Sedlak, T. W. \& Sawa, A. Oxidative stress and schizophrenia: recent breakthroughs from an old story. Curr. Opin. Psychiatry 27, 185-190 (2014).

41. Buonocore, G. \& Perrone, S. Biomarkers of oxidative stress in the fetus and newborn. Haematol. Reps. 2, 103-107 (2006).

42. Ramos, D. et al. Mechanism of copper uptake from blood plasma ceruloplasmin by mammalian cells. PLOS. ONE 11, e0149516 (2016).

43. Abood, L. G., Gibbs, F. A. \& Gibbs, E. Comparative study of blood ceruloplasmin in schizophrenia and other disorders. AMA Arch. Neurol. Psychiatry 77, 643-645 (1957).

44. Puzynski, S. Investigations on ceruloplasmin in chronic schizophrenia. Pol. Med. J. 5, 1484-1491 (1966)

45. Bowman, M. B. \& Lewis, M. S. The copper hypothesis of schizophrenia: a review. Neurosci. Biobehav. Rev. 6, 321-328 (1982).

46. Virit, O. et al. High ceruloplasmin levels are associated with obsessive compulsive disorder: a case control study. Behav. Brain Funct. 4, 52 (2008).

47. Montes, S., Rivera-Mancia, S., Diaz-Ruiz, A., Tristan-Lopez, L. \& Rios, C. Copper and copper proteins in Parkinson's disease. Oxid. Med. Cell. Longev. 2014, 147251 (2014).

48. Bidaki, R. et al. Mismanagement of Wilson's disease as psychotic disorder. Adv. Biomed. Res. 1, 61 (2012)

49. Svetel, M. et al. Neuropsychiatric aspects of treated Wilson's disease. Park. Relat. Disord. 15, 772-775 (2009)

50. Kristiansson, M., Sorman, K., Tekwe, C. \& Calderon-Garciduenas, L. Urban ai pollution, poverty, violence and health--neurological and immunological aspects as mediating factors. Environ. Res. 140, 511-513 (2015).

51. Heyer, D. B. \& Meredith, R. M. Environmental toxicology: sensitive periods of development and neurodevelopmental disorders. Neurotoxicology 58, 23-41 (2017).

52. Knochel, C. et al. Altered apolipoprotein C expression in association with cognition impairments and hippocampus volume in schizophrenia and bipolar disorder. Eur. Arch. Psychiatry Clin. Neurosci. 267 199-212 (2016)

53. Tomasik, J., Rahmoune, H., Guest, P. C. \& Bahn, S. Neuroimmune biomarkers in schizophrenia. Schizophr. Res. 176, 3-13 (2016).

54. Fillman, S. G. et al. Increased inflammatory markers identified in the dorsolateral prefrontal cortex of individuals with schizophrenia. Mol. Psychiatry $\mathbf{1 8}$ 206-214 (2013).

55. Huang, T. L. Decreased serum albumin levels in Taiwanese patients with schizophrenia. Psychiatry Clin. Neurosci. 56, 627-630 (2002).

56. Babushkina, T. A. et al. Study of high-resolution $\mathrm{H} 1$ nuclear magnetic resonance spectra of the serum and its albumin faction in patients with the first schizophrenia episode. Bull. Exp. Biol. Med. 152, 748-751 (2012).

57. Reddy, R., Keshavan, M. \& Yao, J. K. Reduced plasma antioxidants in firstepisode patients with schizophrenia. Schizophr. Res. 62, 205-212 (2003).

58. Viana, J. et al. Schizophrenia-associated methylomic variation: molecular signatures of disease and polygenic risk burden across multiple brain regions. Hum. Mol. Genet. 26, 210-225 (2016).

59. La, Y. J. et al. Decreased levels of apolipoprotein A-l in plasma of schizophrenic patients. J. Neural Transm. 114, 657-663 (2007).

60. Yang, Y. et al. Altered levels of acute phase proteins in the plasma of patients with schizophrenia. Anal. Chem. 78, 3571-3576 (2006).
61. Levin, Y. et al. Global proteomic profiling reveals altered proteomic signature in schizophrenia serum. Mol. Psychiatry 15, 1088-1100 (2010).

62. Jiang, L. et al. Proteomic analysis of the cerebrospinal fluid of patients with schizophrenia. Amino Acids 25, 49-57 (2003)

63. Thomas, E. A., Dean, B., Pavey, G. \& Sutcliffe, J. G. Increased CNS levels of apolipoprotein $D$ in schizophrenic and bipolar subjects: implications for the pathophysiology of psychiatric disorders. Proc. Natl. Acad. Sci. USA 98, 4066-4071 (2001)

64. Mahadik, S. P. Khan, M. M., Evans, D. R. \& Parikh, V. V. Elevated plasma level of apolipoprotein $D$ in schizophrenia and its treatment and outcome. Schizophr. Res. 58, 55-62 (2002).

65. Dean, B. et al. Increased levels of apolipoprotein $E$ in the frontal cortex of subjects with schizophrenia. Biol. Psychiatry 54, 616-622 (2003).

66. Sainz, J. et al. Inflammatory and immune response genes have significantly altered expression in schizophrenia. Mol. Psychiatry 18, 1056-1057 (2013).

67. Wong, C. T., Tsoi, W. F. \& Saha, N. Acute phase proteins in male Chinese schizophrenic patients in Singapore. Schizophr. Res. 22, 165-171 (1996).

68. Wolf, T. L., Kotun, J. \& Meador-Woodruff, J. H. Plasma copper, iron, ceruloplasmin and ferroxidase activity in schizophrenia. Schizophr. Res. 86 167-171 (2006).

69. Shastri, A., Bonifati, D. M. \& Kishore, U. Innate immunity and neuroinflammation. Mediat. Inflamm. 2013, 342931 (2013).

70. Schwarz, E., Guest, P. C., Steiner, J., Bogerts, B. \& Bahn, S. Identification of bloodbased molecular signatures for prediction of response and relapse in schizophrenia patients. Transl. Psychiatry 2, e82 (2012).

71. Athanas, K. M., Mauney, S. L. \& Woo, T. U. Increased extracellular clusterin in the prefrontal cortex in schizophrenia. Schizophr. Res. 169, 381-385 (2015).

72. Jaros J. A., et al. Protein phosphorylation patterns in serum from schizophrenia patients and healthy controls. J. Proteomics. 76, Spec No: 43-55 (2012).

73. Johnson, G. et al. Cerebrospinal fluid protein variations in common to Alzheimer's disease and schizophrenia. Appl. Theor. Electrophor. 3, 47-53 (1992).

74. Maes, M. et al. Haptoglobin polymorphism and schizophrenia: genetic variation on chromosome 16. Psychiatry Res. 104, 1-9 (2001).

75. Rudduck, C., Franzen, G., Frohlander, N. \& Lindstrom, L. Haptoglobin and transferrin types in schizophrenia. Hum. Hered. 35, 65-68 (1985).

76. Saha, N., Bhattacharyya, S. P. \& Tsoi, W. F. Serum protein markers in schizophrenia: haptoglobin, transferrin and group-specific component types. Singap. Med. J. 26, 337-340 (1985).

77. Pandey, J. P., Namboodiri, A. M. \& Elston, R. C. Immunoglobulin G genotypes and the risk of schizophrenia. Hum. Genet. 135, 1175-1179 (2016).

78. Blomstrom, A., Gardner, R. M., Dalman, C., Yolken, R. H. \& Karlsson, H. Influence of maternal infections on neonatal acute phase proteins and their interaction in the development of non-affective psychosis. Transl. Psychiatry 5, e502 (2015).

79. Schizophrenia Psychiatric Genome-Wide Association Study C. Genome-wide association study identifies five new schizophrenia loci. Nat. Genet. 43, 969-976 (2011).

80. Herberth, M. et al. Impaired glycolytic response in peripheral blood mononuclear cells of first-onset antipsychotic-naive schizophrenia patients. Mol. Psychiatry 16, 848-859 (2011).

81. Yao, J. K et al. Homeostatic imbalance of purine catabolism in first-episode neuroleptic-naive patients with schizophrenia. PLoS. ONE 5, e9508 (2010).

82. Lawrence, T. \& Natoli, G. Transcriptional regulation of macrophage polarization: enabling diversity with identity. Nat. Rev. Immunol. 11, 750-761 (2011).

83. Saia-Cereda, V. M. et al. Proteomics of the corpus callosum unravel pivotal players in the dysfunction of cell signaling, structure, and myelination in schizophrenia brains. Eur. Arch. Psychiatry Clin. Neurosci. 265, 601-612 (2015). 